\title{
Solving Suggested Problems using Grey Wolf Optimization
}

\author{
Nawras A. Issa ${ }^{1}$, Zaid Alaa ${ }^{2}$, and Issa Ahmed Abed ${ }^{3}$ \\ \{nwrsb372@gmail.com ${ }^{1}$, zaid.alaa@stu.edu.iq ${ }^{2}$, issaahmedabed@stu.edu.iq ${ }^{3}$ \} \\ Basrah Engineering Technical College, Southern Technical University, Iraq ${ }^{1}$ \\ Management Technical College - Basra, Southern Technical University, Iraq ${ }^{2,3}$
}

\begin{abstract}
In this research, the Grey Wolf Optimizer (GWO) algorithm was applied, which is a type of metaheuristic optimization algorithms that is important for identifying problems and finding ways to improve them. In 2014, Seyedali Mirjalili came up with this algorithm. Its social hierarchy is modeled after that of grey wolves in nature. These wolves live in groups consisting of $(5-12)$ individuals. Wolves are divided into four levels, alpha represents the first level it is accountable for the manufacture of important resolutions for the peak like hunting, bedtime, wake up, etc. As for the second level in the hierarchy, it represents the beta wolf and is the advisor of the alpha. Beta can be the leader after the death of one of the alpha wolves. The third level represents delta, which follows the commands of alpha and beta. it is the dominant omega. omega represents the last level, which obeys all other wolves. Furthermore, the chief algorithm stages such as chasing, searching the prey, encircle and attack the prey were applied. GWO algorithm was tested on three benchmarks test functions using MATLAB R2014a. the results were confirmed by comparing GWO through another intelligent swarm algorithm like Particle Swarm Optimization (PSO) algorithm. results showed the superiority GWO in achieving better results and high convergence speed.
\end{abstract}

Keywords: Metaheuristic Algorithm, Grey Wolfe Optimizer.

\section{Introduction}

optimization is getting the best result under certain conditions like minimum costs, maximize efficiency or maximize revenues. Some optimization strategies have been created that are conceptually different from traditional techniques. Modern optimization approaches are based on biological, molecular, insect swarm, and neurobiological systems features and behavior[1]. With the advancement of information technology, a large number of optimization problems develop in a variety of disciplines like Internet routing, economics, and engineering design, which can be solved using a variety of ways such as, Fuzzy Logic[2],[3]. Sine Cosine Algorithm(SCA)[4]. ant colony optimization (ACO)[5], PSO algorithm [6], and so on.

Meta-heuristic algorithms are more reliable and usable by researchers in different fields rather than traditional optimization algorithms. Because these algorithms are population-based stochastic algorithms, their solution quality is far superior to that of traditional optimization techniques because of fewer parameters, high convergence speed, simplicity implementation [7]. It can be divided into three categories: i) Evolutionary Algorithms (EA), like Genetic Algorithm (GA)[8], A Differential Evolution Algorithm (DE)[9]. ii) physics-based algorithms, like gravitational search algorithm (GSA)[10],Harmony Search Algorithm[11] and Simulated Annealing[12].iii) Swarm intelligence (SI)-based algorithm, like Particle Swarm (PSO)[13], 
Ant Colony Optimization(ACO)[14], Artificial Bee Colony[15], Fish Swarm Optimization(FSA)[16], Cuckoo Search algorithm (CS)[17], Bat Algorithm(BA)[18], Firefly Algorithm(FA)[19],[20]. and grey wolf optimization (GWO)[21]. GWO is inspired by grey wolves in nature, who are always looking for the best approach to catch their prey. In nature, the GWO algorithm uses the same process to organize the various functions in the wolf pack, which follows the pack hierarchy [22]. Pack's members are divided into four levels. alpha, beta, delta, and last level omega. it is best among the intelligent swarm algorithms because of simple, straightforward to use, and scalable, with a unique capacity to find the correct equilibrium between exploration (searching) and exploitation (attacking) in the search [23],[24]. GWO uses a small number of parameters to solve non-deterministic polynomial (NP)-hard problems over several rounds [25]. As a result, it was used in a variety of applications see figure 1.

(GWO) was used in different optimization processes such as the hybrid power generation system's optimal design to the less total cost.[26].to determine the best operating strategy for economic load dispatch (ELD) [27]. to solve the challenge of robot path planning to find the shortest path [28]. In WSNs, used for cluster head selection [29]. to achieve a stable network and have high energy-efficient [30]. In the industrial world, optimizing fuel use is the most significant concern. Several causes have played a role in the recent rise in relevance. Increasing energy consumption and dwindling fossil fuel supplies are two of these factors. Instead of fossil fuels, nuclear energy can be used in most power-producing systems. Because nuclear fuel production is such a complicated process, it's critical to think about how it's used. based on neutronic and thermal-hydraulic parameters, to establish the optimal pattern for the core of the Bushehr nuclear power station [31]. The optimal parameters of a PID controller for load frequency regulation in a multi-source single area power system were estimated using the GWO algorithm, which is based on evolutionary intelligence. The power grid is made up of thermal, gas, and hydroelectric power facilities with mechanical hydraulic governors [32]. To minimize load bus voltage variations and system power losses, the grey wolf optimizer (GWO) was used to determine the appropriate placement of STATCOM devices on the power system grid. [33]. Controlling electrical machinery with the DTC method is common. To match the speed to the specified reference, the parameters of the conventional Proportional Integral (PI) speed controller are manually changed. The grey wolf algorithm(GWO) is used to modify the PI speed controller parameters to improved Direct Torque Control (DTC) and to improve the performance of the Induction Motor (IM)[34]. The GWO algorithm is implemented. The main goals of this report are i) study the GWO algorithm to identify problems and find the best solutions. ii) comparison of the GWO with PSO algorithm. iii) simulation of the algorithm using MATLA environment. The results proved the efficiency of GWO in finding the optimal solution, high convergence speed, and avoiding local optimization.

\section{Grey Wolf Optimization (GWO)}

GWO is population-based swarm intelligence. That imitates grey wolf dominance. Mirjalili had proposed it in 2014. Grey wolves are social animals that live in packs of 5 to 12 individuals. for each iteration, the three best probable solutions are known to the alpha wolf, beta wolf, and delta wolf they lead to wide areas of the search. The dominant hierarchy in the group is divided into four levels [25],[26] as shown in Figure 2. this algorithm has been employed by several academics due to its simplicity and ease of use. The grey wolf optimization (GWO) uses a few numbers of parameters to solve non-deterministic polynomial (NP)-hard problems over several iterations. 


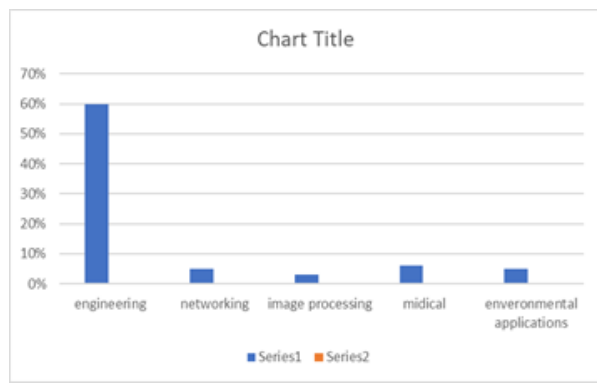

Fig 1. GWO application.

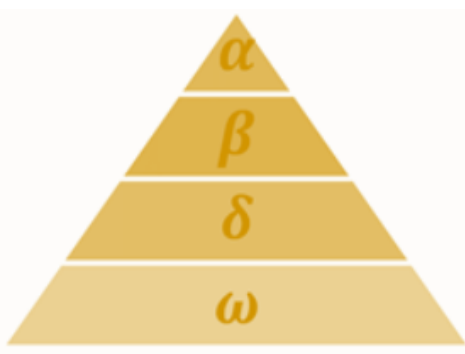

Fig 2. Grey wolf social hierarchy.

\subsection{Social Hierarchy}

Alpha wolf $(\alpha)$, who can be male or female, is the group's leader. It is the pack's highest-ranking member, in charge of hunting, sleeping arrangements, and walking schedules. Beta $(\beta)$ The second type of wolves in the pack, which help the alpha in their decisions about days activities. the third level in the dominant social hierarchy is called delta wolf $(\delta)$ and submits to alpha and beta members. Omega wolf $(\omega)$ has the last hierarchical rank in the group and submits to all the other wolves, but it plays a critical role in balancing hierarchy [35].

\subsubsection{Encircling Process}

Grey wolves surround their victim as part of the hunting phase, see figure 3 . the following equations are used to quantitatively model encircling behavior mathematically as shown below [36].

$|\vec{D}=| \vec{C} \cdot \vec{X}_{p}(t)-\vec{X}(t) \mid$

$\vec{X}(t+1)=\vec{X}_{p}(t)-\vec{A} \cdot \vec{D}$

when $(\mathrm{t})$ refers to the current iteration, $\left(\mathrm{A}^{\rightarrow}\right)$ and $\left(\mathrm{C}^{\rightarrow}\right)$ represents the coefficient vectors see equations (3) and (4). (Xp) $\rightarrow$ indicates the location vector of the prey, and $(\mathrm{X} \rightarrow$ ) refers to the location vector of a grey wolf [36].

$\vec{A}=2 \vec{a} \cdot \vec{r}_{1}-\vec{a}$

$\vec{C}=2 \cdot \vec{r}_{2}$

When (r_1 ) $\rightarrow$ and (r_2 ) $\rightarrow$ represented to random vectors in the range $[0,1]$ so the component of $\mathrm{a} \rightarrow$ is reduced linearly from $(2-0)$ across repeated iterations. the grey wolves update positions for (2D) and (3D) are shown in Figure 4. ( $a$ and b). 

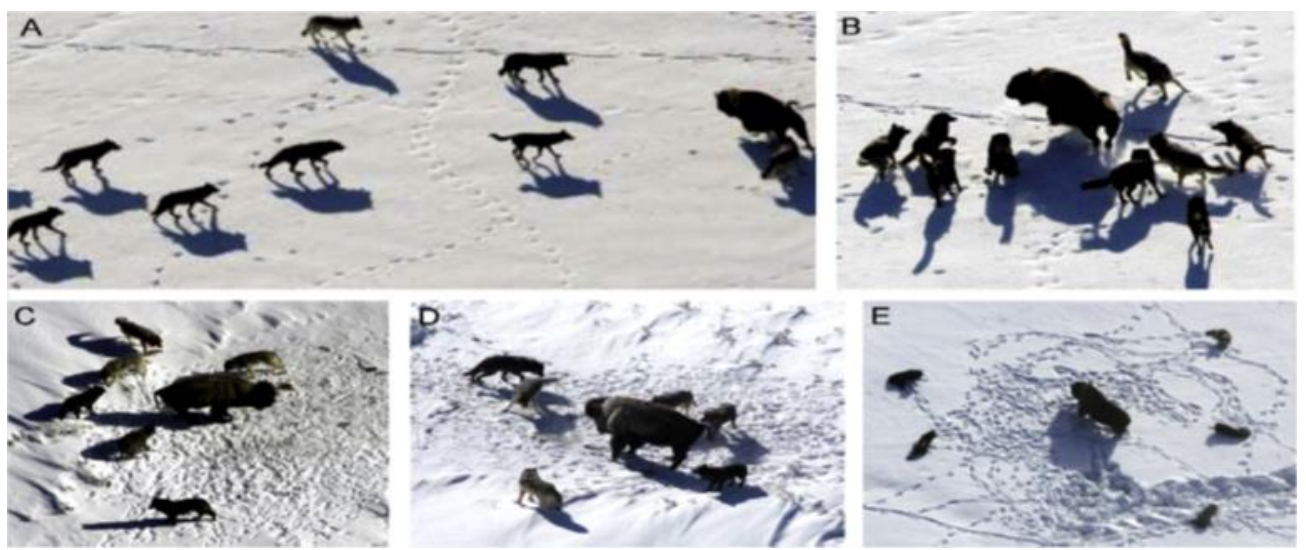

Fig 3. Grey wolf hunting behavior: (A) chasing, impending, and following victim(B-D) troublesome, and surrounding (E)attack and fixed state.

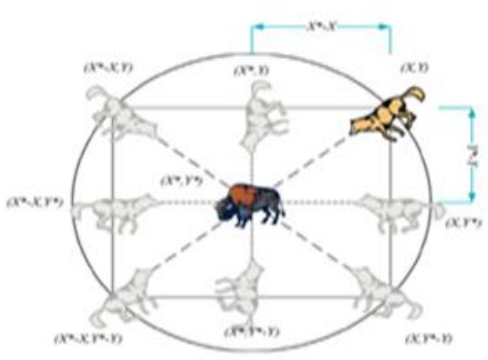

(a)

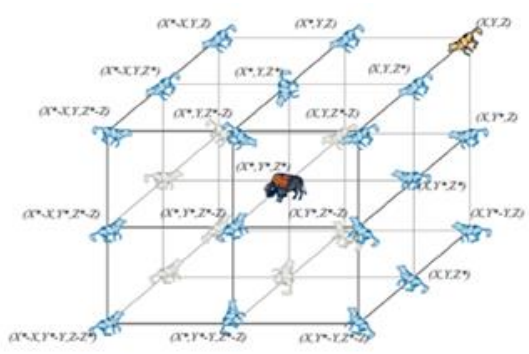

(b)

Fig 4. (a) 2 dimensions position vectors, (b)3 dimintions position vectors.

\subsubsection{Hunting Process}

The leader (alpha) guides the other wolves (beta )and (delta) through the procedure for hunting. in the mathematical model, the alpha represents the overall best solutions, while beta $(\beta)$ and $\operatorname{delta}(\delta)$ refer to the second and third best candidate solutions respectively in the population. All solutions are updated their positions according to the location of the best first three solutions (alpha $(\alpha), \operatorname{beta}(\beta)$, and delta $(\delta)$ successively $)[37]$. The formula of the mathematical equation of the hunting process is shown below.

$$
\begin{aligned}
\vec{D}_{\alpha} & =\left|\vec{C}_{1} \cdot \vec{X}_{\alpha}-\vec{X}\right| \\
\vec{D}_{\beta} & =\left|\vec{C}_{2} \cdot \vec{X}_{\beta}-\vec{X}\right| \\
\vec{D}_{\delta} & =\left|\vec{C}_{3} \cdot \vec{X}_{\delta}-\vec{X}\right| \\
\vec{X}_{1} & =\vec{X}_{\alpha}-\vec{A}_{1} \cdot\left(\vec{D}_{\alpha}\right)
\end{aligned}
$$




$$
\begin{aligned}
& \vec{X}_{2}=\vec{X}_{\beta}-\vec{A}_{2} \cdot\left(\vec{D}_{\beta}\right) \\
& \vec{X}_{3}=\vec{X}_{\delta}-\vec{A}_{3} \cdot\left(\vec{D}_{\delta}\right) \\
& \vec{X}(t+1)=\frac{\vec{X}_{1}+\vec{X}_{2}+\vec{X}_{3}}{3}
\end{aligned}
$$

When $\left(\mathrm{X}^{\rightarrow} 1\right),\left(\mathrm{X}_{-}{ }_{-}\right)$, and $\left(\mathrm{X}^{\rightarrow} 3\right)$ refer to the first three solutions in the population. Figure 5. A search agent's position is updated based on alpha, beta, and delta coordinates. In the context of a two-dimensional search space.

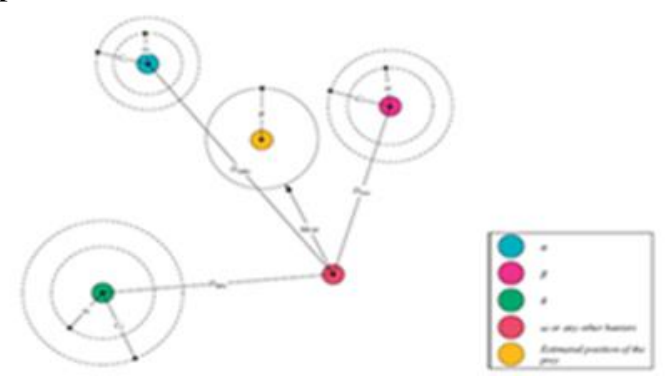

Fig 5. Position updating to grey wolves.

\subsubsection{Attacking Process (Exploitation)}

The hunt is completed when the prey is attacked. When the vector( A )is a random number in the interval [ $-2 \mathrm{a}, 2 \mathrm{a}]$ and a decreased liberty from ( 2 to 0 ) throughout iterations, the wolves progress towards the prey. figure $6($ a) demonstrates that $|\mathrm{A}|<1$ makes the wolves attack prey. [36],[37].

\subsubsection{Search Process for Prey (Exploration)}

Each wolf modifies its location dependent on alpha and data's positions to hunt for prey. Diversification or exploration is the term for this procedure. When $|\mathrm{A}|>1$, the wolves (solutions) are driven to leave the prey in search of more suitable prey. Figure 6 (b) indicates that when $|\mathrm{A}|$ $>1$ grey wolves are forced to diverge from their prey in the hopes of finding a fitter prey. $\mathrm{C}$ is an additional component that aids the algorithm in finding new solutions. when $\mathrm{C}>1$ emphasize or $\mathrm{C}<1$ deemphasize random weights for the prey [36],[37].

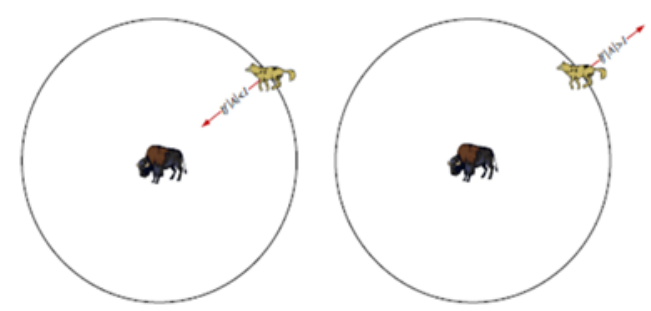

Fig 6. (a) Attacking prey,(b) searching for prey. 


\subsection{Optimization Processing and Pseudo-Code to GWO Algorithim}

The GWO optimization method begins with a randomly generated grey wolf population (candidate solutions). Throughout the iterations, the wolves' alpha, beta, and delta assess the prey's likely location (optimum solution). The distance between grey wolves and their prey determines how they position themselves. Parameter a should be reduced from 2 to 0 to emphasize the exploration process and exploitation process during the search phase [38]. The pseudo-code is represented in Fig 7.

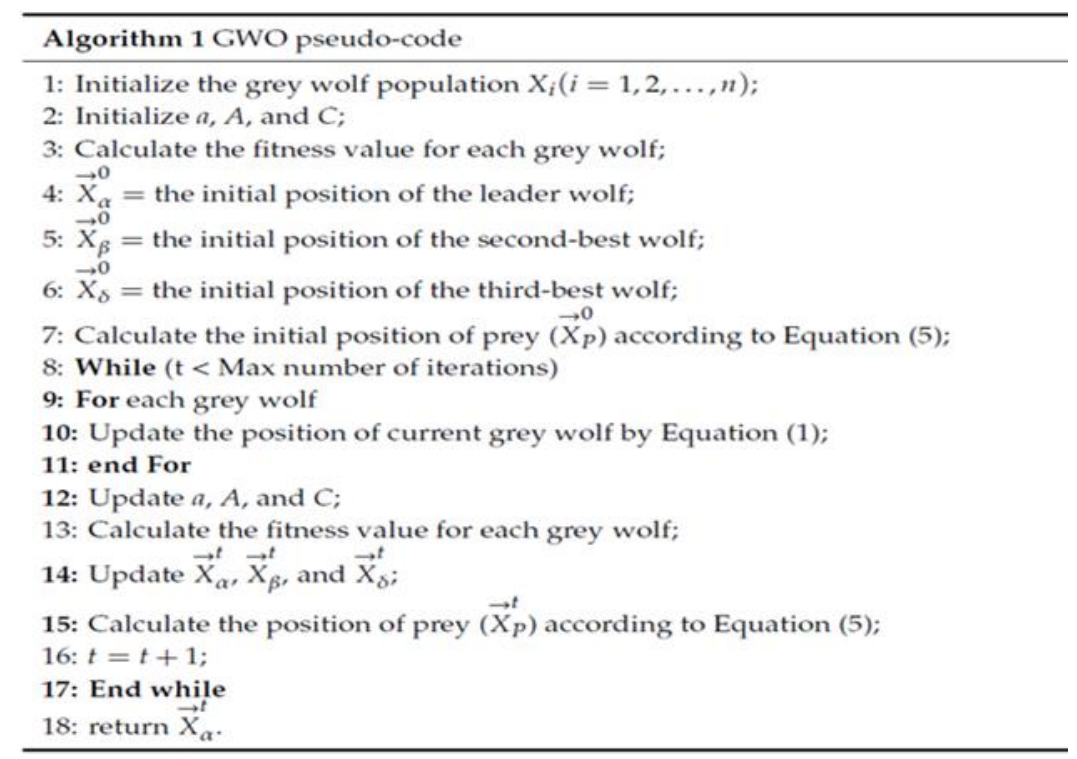

Fig 7. Pseudo-code of GWO algorithm[39].

\section{Simulation Results and Comparison}

The GWO was implemented in MATLAB R2014a.Laptop specifications, device name DESKTOP-14BPU59,Processor Intel(R) Core(TM) i7-8565U CPU @ $1.80 \mathrm{GHz} \quad 1.99$ GHz,Installed RAM 8.00 GB (7.88 GB usable), System type 64-bit operating system. Windows 10 Pro.

We employ three benchmark test functions[36],[40] to compare the proposed GWO algorithm against other population-based approaches to assess its performance. Table 1 shows these test functions. the GWO method was compared to the PSO algorithm. with the same setting of the parameters, that is, dimension ( numbers of variables ) is set to 4 , the size of the population is set 30 , and numbers of maximum iterations $(30,90,150)$ for all test functions. we tested each function 10 times. each algorithm was performed ten times to find the best solution, average value and standard deviation of extracted results were evaluated. Table 2 shows the first case ( max.iter.=30), GWO gives the better results ( best, mean) for all functions f1, f2, f3.also the standard deviation of GWO is less than the PSO algorithm. when the standard deviation is less, the GWO is stable. Table 3 shows the second case (max.iter.=90) when the iteration process is 
increased, GWO gives better results compared to the first case for all functions. Table 4 shows the third case ( max.iter.=150). when iteration increases the advantages of GWO to find the best solution, the mean increases too, compared to PSO. the best value is close to the optimal value and the standard deviation is as little as possible, which makes the GWO more stable in optimization operations. Figure 8(a,b,c), Figure 9(a,b,c) and Figure 10(a,b,c) illustrate the convergence curves of fitness values with deference to the number of maximum iterations for the 3 functions to test respectively. The GWO algorithm is more effective than the PSO algorithm in reaching the optimal solution for all test functions under the same conditions as iteration increases.

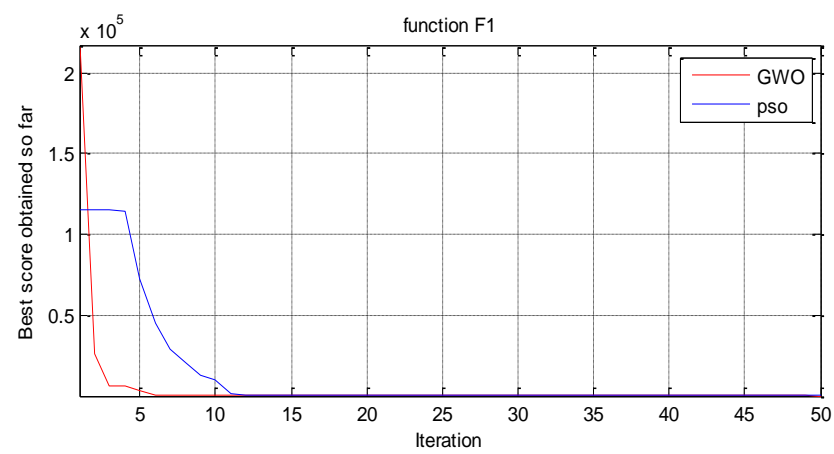

(a)

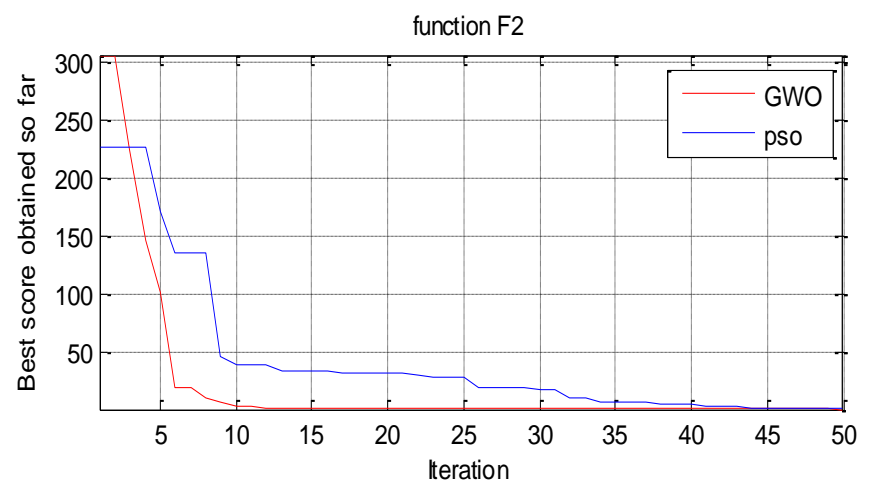

(b) 


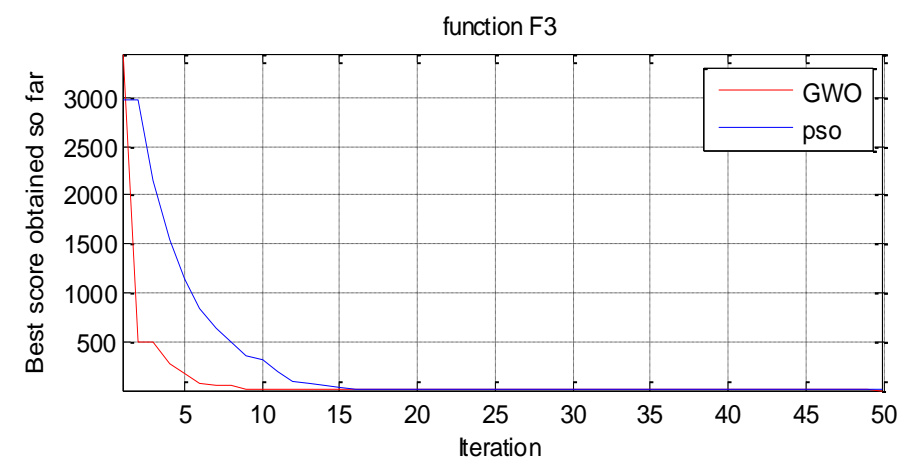

(c)

Fig 8. (a,b,c). convergence curve of GWO ana PSO with maximum iteration 50 for 3 test functions $\mathrm{f} 1, \mathrm{f} 2$, and $\mathrm{f} 3$.

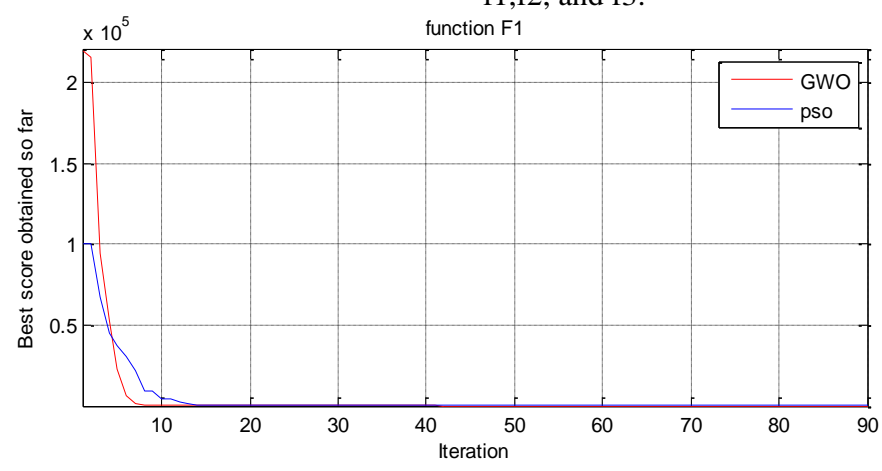

(a)

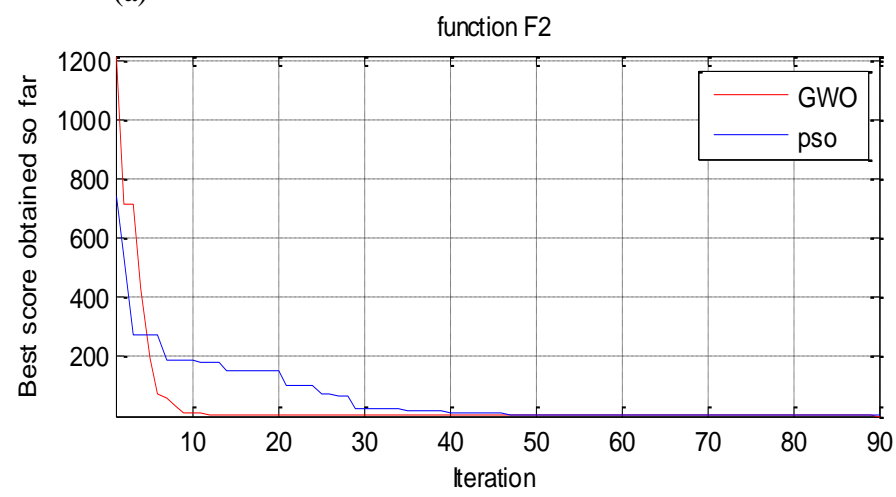

(b) 


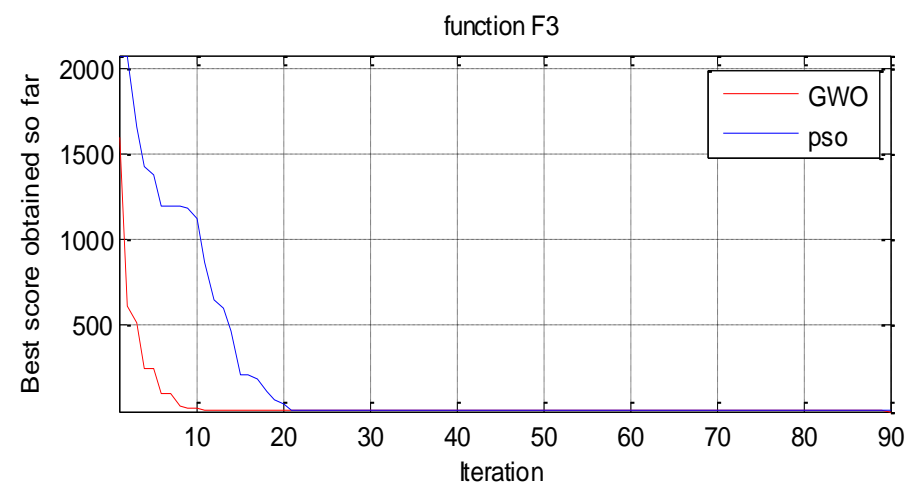

(c)

Fig 9. $(a, b, c)$. convergence curve of GWO ana PSO with maximum iteration 90 for 3 test functions $\mathrm{f} 1, \mathrm{f} 2$, and $\mathrm{f} 3$.

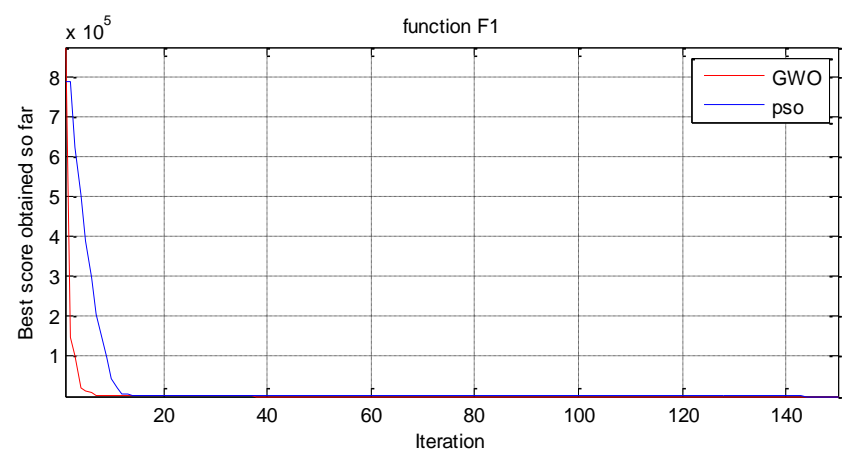

(a)

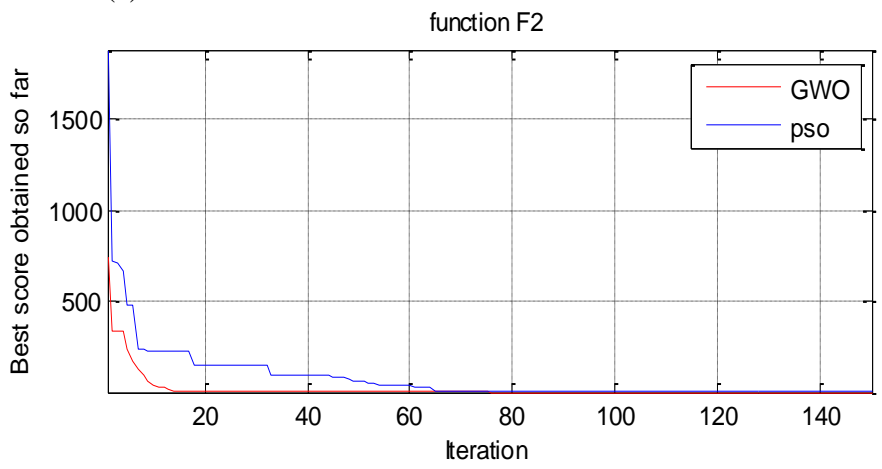

(b) 


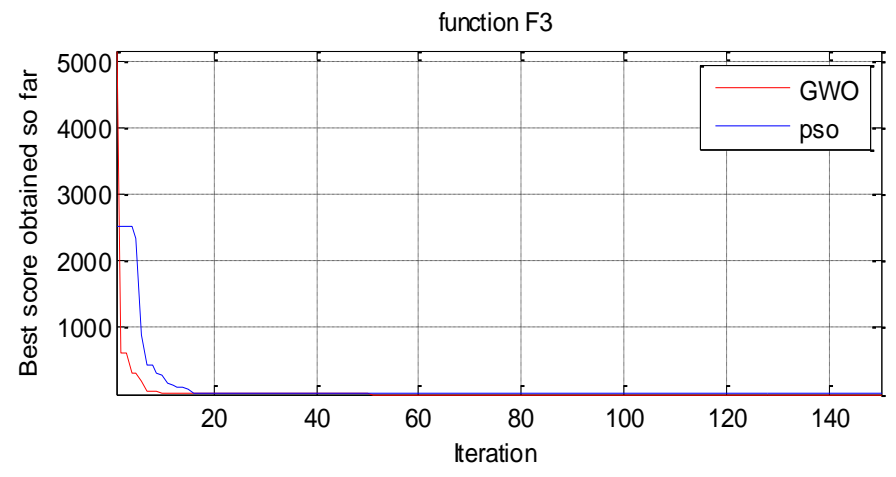

(c)

Figure 10. Convergence curve of GWO ana PSO with maximum iteration 150 for 3 test functions f1,f2, and $\mathrm{f} 3$.

Table 1. Unimodel benchmark test functions ( standard optimization functions).

\begin{tabular}{|lcccccc|}
\hline Function & Pop. zize max.Iter. & Dim. & Rang & min \\
\hline$f_{1}(x)=\sum_{i=1}^{n} x_{i}^{2}$ & 30 & $(30,90,150)$ & 4 & {$[-100,100]$} & 0 \\
$f_{2}(x)=\sum_{i=1}^{n}\left|x_{i}\right|+\prod_{i=1}^{n}\left|x_{i}\right|$ & 30 & $(30,90,150)$ & 4 & {$[-10,10]$} & 0 \\
$f_{3}(x)=\sum_{i=1}^{n}\left(\sum_{j-1}^{i} x_{j}\right)^{2}$ & 30 & $(30,90,150)$ & 4 & {$[-100,100]$} & 0 \\
\hline
\end{tabular}

Table 2. Experimental results comparison of GWO and PSO with max.Iteration 30 and run 10 for 3 test functions.

\begin{tabular}{|lcccc|}
\hline Function & Algorithm & Best values & Mean values & St.dev. \\
\hline \multirow{2}{*}{ F1 } & GWO & $1.84 \mathrm{E}-06$ & $1.08 \mathrm{E}-03$ & 0.002935557 \\
& PSO & $1.81 \mathrm{E}-02$ & $2.63 \mathrm{E}-01$ & 0.273849719 \\
F2 & & & \\
& GWO & 0.0013555 & 0.01357527 & 0.014878658 \\
F3 & PSO & 0.8733 & 2.09849 & 1.309364767 \\
& GWO & 0.0015572 & 0.06622358 & 0.113190574 \\
& PSO & 0.0085606 & 0.06569306 & 0.067863718 \\
\hline
\end{tabular}


Table 3. Experimental results comparison of GWO and PSO with max.Iteration 90 and run 10 for 3 test functions.

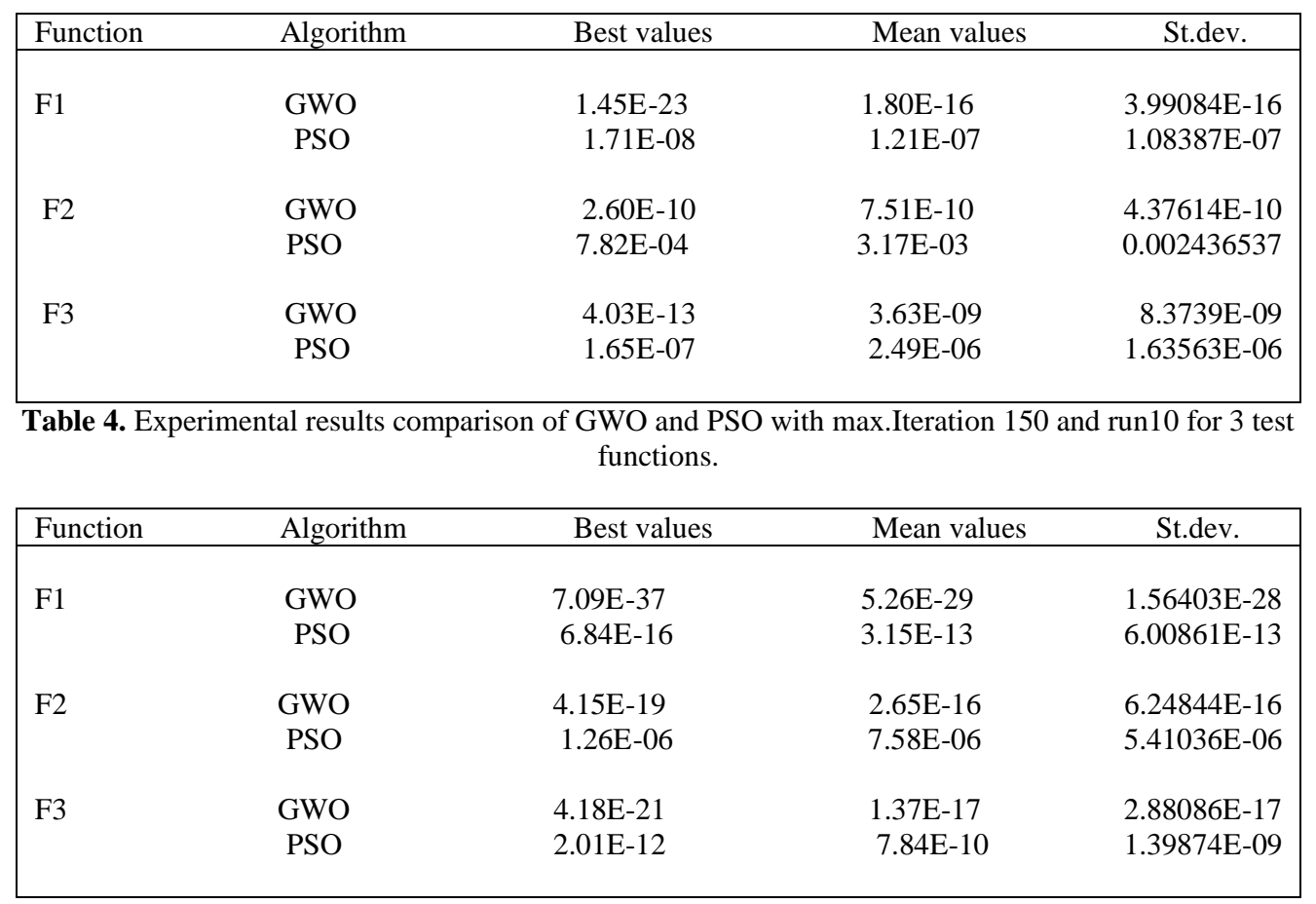

\section{Conclusions}

This paper defined the grey wolf optimizer (GWO) algorithm, which is a meta-heuristic algorithm that is quite recent. as it depends on population diversity to discover the optimum solution and achieving a balance between the exploration and exploitation process. GWO algorithm is inspired by the hunting behavior of the grey wolves in nature(hunting mechanisms and social hierarchy). As well, this research included mathematical models for encircling, tracking, and hunting. to verify the results of the GWO algorithm, three test benchmark functions were used to compare with another algorithm like PSO with the same conditions. the simulation results obtained using MATLAB R2014a environment proved the ability of GWO to achieve faster and better results access the optimal global solution and escaping local solutions.

\section{References}

[1] K. Suresh, G. V. Kumar, and K. N. Raju, "Cost optimization for intelligent enterprises using modified GWO,” Proc. 3rd Int. Conf. Smart Syst. Inven. Technol. ICSSIT 2020, no. Icssit, pp. 87-91, 2020, doi: 10.1109/ICSSIT48917.2020.9214166.

[2] Y. Tao, J. Zhang, and L. Yang, "An unequal clustering algorithm for wireless sensor networks based on interval type-2 tsk fuzzy logic theory,” IEEE Access, vol. 8, pp. 197173-197183, 2020, doi: 10.1109/ACCESS.2020.3034607. 
[3] M. Mirsadeghi, A. Mahani, and M. Shojaee, "A Novel Distributed Clustering Protocol Using Fuzzy Logic," Procedia Technol., vol. 17, pp. 742-748, 2014, doi: 10.1016/j.protcy.2014.10.193.

[4] S. Mirjalili, "SCA: A Sine Cosine Algorithm for solving optimization problems," KnowledgeBased Syst., vol. 96, pp. 120-133, 2016, doi: 10.1016/j.knosys.2015.12.022.

[5] C. Shin and M. Lee, "Swarm-intelligence-centric routing algorithm for wireless sensor networks," Sensors (Switzerland), vol. 20, no. 18, pp. 1-13, 2020, doi: 10.3390/s20185164.

[6] M. Ren, X. Huang, X. Zhu, and L. Shao, "Optimized PSO algorithm based on the simplicial algorithm of fixed point theory," Appl. Intell., vol. 50, no. 7, pp. 2009-2024, 2020, doi: 10.1007/s10489-020-01630-6.

[7] M. Abdel-Basset, L. Abdel-Fatah, and A. K. Sangaiah, Metaheuristic algorithms: A comprehensive review. Elsevier Inc., 2018.

[8] S. Chen, T. R. Newman, J. B. Evans, and A. M. Wyglinski, "Genetic algorithm-based optimization for cognitive radio networks,” 33rd IEEE Sarnoff Symp. 2010, Conf. Proc., 2010, doi: 10.1109/SARNOF.2010.5469780.

[9] R. Knobloch, "A differential evolution algorithm in the optimization task with a Lipschitz continuous cost function," AIP Conf. Proc., vol. 2048, no. December, 2018, doi: $10.1063 / 1.5082064$.

[10] H. A. Kherabadi, S. E. Mood, and M. M. Javidi, "Mutation: A new operator in gravitational search algorithm using fuzzy controller,” Cybern. Inf. Technol., vol. 17, no. 1, pp. 72-86, 2017, doi: 10.1515/cait-2017-0006.

[11] A. Askarzadeh and E. Rashedi, Harmony search algorithm: Basic concepts and engineering applications, no. April. 2017.

[12] J. Zeng, Z. Huang, Y. Chen, W. Liu, and S. Chu, "A simulated annealing approach for optimizing composite structures blended with multiple stacking sequence tables," Struct. Multidiscip. Optim., vol. 60, no. 2, pp. 537-563, 2019, doi: 10.1007/s00158-019-02223-9.

[13] D. Wang, D. Tan, and L. Liu, "Particle swarm optimization algorithm: an overview," Soft Comput., vol. 22, no. 2, pp. 387-408, 2018, doi: 10.1007/s00500-016-2474-6.

[14] A. S. Pontes et al., "Ant colony optimization for variable selection in discriminant linear analysis," J. Chemom., vol. 34, no. 12, pp. 1-12, 2020, doi: 10.1002/cem.3292.

[15] B. Crawford, R. Soto, R. Cuesta, and F. Paredes, "Application of the artificial bee colony algorithm for solving the set covering problem," Sci. World J., vol. 2014, 2014, doi: $10.1155 / 2014 / 189164$.

[16] C. Zhang, F. M. Zhang, F. Li, and H. S. Wu, "Improved artificial fish swarm algorithm," Proc. 2014 9th IEEE Conf. Ind. Electron. Appl. ICIEA 2014, vol. 11, pp. 748-753, 2014, doi: 10.1109/ICIEA.2014.6931262.

[17] A. S. Joshi, O. Kulkarni, G. M. Kakandikar, and V. M. Nandedkar, "Cuckoo Search Optimization- A Review," Mater. Today Proc., vol. 4, no. 8, pp. 7262-7269, 2017, doi: 10.1016/j.matpr.2017.07.055.

[18] M. A. Al-Betar and M. A. Awadallah, "Island bat algorithm for optimization," Expert Syst. Appl., vol. 107, pp. 126-145, 2018, doi: 10.1016/j.eswa.2018.04.024.

[19] N. Farahlina Johari, A. Mohd Zain, N. Haszlinna Mustaffa, and A. Udin, "Machining Parameters Optimization using Hybrid Firefly Algorithm and Particle Swarm Optimization," J. Phys. Conf. Ser., vol. 892, no. 1, pp. 0-11, 2017, doi: 10.1088/1742-6596/892/1/012005.

[20] W. A. Hasan, I. A. Abed, and D. K. Shary, "Management of Microgrid System Based on Optimization Algorithm," Journal of Physics: Conference Series, vol. 1773, no. 1. 2021, doi: 10.1088/1742-6596/1773/1/012006.

[21] S. Saremi, S. Z. Mirjalili, and S. M. Mirjalili, "Evolutionary population dynamics and grey wolf optimizer," Neural Comput. Appl., vol. 26, no. 5, pp. 1257-1263, 2015, doi: 10.1007/s00521014-1806-7.

[22] N. Jayakumar, S. Subramanian, S. Ganesan, and E. B. Elanchezhian, "Grey wolf optimization for combined heat and power dispatch with cogeneration systems," Int. J. Electr. Power Energy Syst., vol. 74, pp. 252-264, 2016, doi: 10.1016/j.ijepes.2015.07.031. 
[23] A. Al-Moalmi, J. Luo, A. Salah, and K. Li, "Optimal virtual machine placement based on grey wolf optimization," Electron., vol. 8, no. 3, pp. 1-22, 2019, doi: 10.3390/electronics8030283.

[24] S. Y. Li, S. M. Wang, P. F. Wang, X. L. Su, X. S. Zhang, and Z. H. Dong, "An improved grey wolf optimizer algorithm for the inversion of geoelectrical data," Acta Geophys., vol. 66, no. 4, pp. 607-621, 2018, doi: 10.1007/s11600-018-0148-8.

[25] A. Farshin and S. Sharifian, "A chaotic grey wolf controller allocator for Software Defined Mobile Network (SDMN) for 5th generation of cloud-based cellular systems (5G)," Comput. Commun., vol. 108, pp. 94-109, 2017, doi: 10.1016/j.comcom.2017.05.003.

[26] A. Yahiaoui, F. Fodhil, K. Benmansour, M. Tadjine, and N. Cheggaga, "Grey wolf optimizer for optimal design of hybrid renewable energy system PV-Diesel Generator-Battery: Application to the case of Djanet city of Algeria," Sol. Energy, vol. 158, no. August, pp. 941951, 2017, doi: 10.1016/j.solener.2017.10.040.

[27] M. Pradhan, P. K. Roy, and T. Pal, "Grey wolf optimization applied to economic load dispatch problems,” Int. J. Electr. Power Energy Syst., vol. 83, pp. 325-334, 2016, doi: 10.1016/j.ijepes.2016.04.034.

[28] T. F. Abaas and A. H. Shabeeb, "Safe and optimum navigation of wheeled mobile robot using grey wolf optimization algorithm," IOP Conf. Ser. Mater. Sci. Eng., vol. 928, no. 2, 2020, doi: 10.1088/1757-899X/928/2/022006.

[29] D. Agrawal et al., "GWO-C: Grey wolf optimizer-based clustering scheme for WSNs," Int. J. Commun. Syst., vol. 33, no. 8, pp. 1-15, 2020, doi: 10.1002/dac.4344.

[30] A. Kaushik, S. Indu, and D. Gupta, "A Grey Wolf Optimization Approach for Improving the Performance of Wireless Sensor Networks,” Wirel. Pers. Commun., no. 0123456789, 2019, doi: 10.1007/s11277-019-06223-2.

[31] A. Naserbegi, M. Aghaie, and A. Zolfaghari, "Implementation of Grey Wolf Optimization (GWO) algorithm to multi-objective loading pattern optimization of a PWR reactor," Ann. Nucl. Energy, vol. 148, no. xxxx, p. 107703, 2020, doi: 10.1016/j.anucene.2020.107703.

[32] N. Paliwal, L. Srivastava, and M. Pandit, "Application of grey wolf optimization algorithm for load frequency control in multi-source single area power system," Evol. Intell., no. 0123456789, 2020, doi: 10.1007/s12065-020-00530-5.

[33] A. A. M. El-Gaafary, Y. S. Mohamed, A. M. Hemeida, and A.-A. A. Mohamed, "Grey Wolf Optimization for Multi Input Multi Output System," Univers. J. Commun. Netw., vol. 3, no. 1, pp. 1-6, 2015, doi: 10.13189/ujen.2015.030101.

[34] G. M. Fadhil, I. A. Abed, and R. S. Jasim, "Controlling of Induction Motor Using Grey Wolf Optimization Algorithm," J. Phys. Conf. Ser., vol. 1773, no. 1, 2021, doi: 10.1088/17426596/1773/1/012007.

[35] J. Too, A. R. Abdullah, N. M. Saad, N. M. Ali, and W. Tee, "A new competitive binary grey wolf optimizer to solve the feature selection problem in EMG signals classification," Computers, vol. 7, no. 4, 2018, doi: 10.3390/computers7040058.

[36] S. Mirjalili, S. M. Mirjalili, and A. Lewis, "Grey Wolf Optimizer," Adv. Eng. Softw., $\quad$ vol. 69, pp. 46-61, 2014, doi: 10.1016/j.advengsoft.2013.12.007.

[37] H. Joshi and S. Arora, "Enhanced grey Wolf optimization algorithm for global optimization," Fundam. Informaticae, vol. 153, no. 3, pp. 235-264, 2017, doi: 10.3233/FI-2017-1539.

[38] Z. Wang, H. Xie, Z. Hu, D. Li, J. Wang, and W. Liang, "Node coverage optimization algorithm for wireless sensor networks based on improved grey wolf optimizer," J. Algorithms Comput. Technol., vol. 13, 2019, doi: 10.1177/1748302619889498.

[39] X. Zhao, S. Ren, H. Quan, and Q. Gao, "Routing protocol for heterogeneous wireless sensor networks based on a modified grey wolf optimizer," Sensors (Switzerland), vol. 20, no. 3, pp. 1-18, 2020, doi: 10.3390/s20030820.

[40] Abed, I. A., S. P. Koh, K. S. M. Sahari, S. K. Tiong, M. M. Ali, and AA Abdul Kadhim. "A New Proposed Pendulum-Like with Attraction-Repulsion mechanism Algorithm 
for Solving Optimization Problems." In IOP Conference Series: Materials Science and Engineering, vol. 881, no. 1, p. 012132. IOP Publishing, 2020. 